\title{
Análisis del comercio electrónico y su aporte en las transacciones internacionales en la región Junín, Perú
}

\author{
Analysis of electronic commerce and its contribution in international \\ transactions in the Junín region, Perú
}

\author{
Jose Maria Matas ${ }^{1,}$, , Miguel Jesús Medina Viruel ${ }^{2}$, Domingo Fernández Uclés ${ }^{2}$ Dayana S. Aguilar García ${ }^{1}$ \\ ${ }^{1}$ Universidad Continental - Perú ${ }^{2}$ Universidad de Jaen - España
}

\section{RESUMEN}

La investigación sobre el análisis del comercio electrónico en la región Junín y su aporte en las transacciones internacionales tiene como objetivo determinar las características de las empresas exportadoras de la región Junín que han contribuido a la implantación de las Tecnologías de la Información y Comunicación y el comercio electrónico. El estudio se desarrolló bajo el tipo de investigación aplicada, con información del directorio de empresas de la región Junín facilitada por la Comisión de Promoción del Perú para la Exportación y el Turismo (Promperú exportaciones- Oficina Macro Regional de Exportación Centro). El nivel de investigación fue descriptivo de corte transversal. El estudio tuvo como objeto de estudio a una población conformado por 104 empresas, de las cuales 35 pertenecen al sector vestimenta y decoración y, 69 al sector agroindustria; éstas a su vez realizan de manera directa e indirecta actividad comercial en el exterior; el tamaño de muestra fue de 62 empresas con un error muestral aproximado de 8,11 por 100, para $p=q=0,5$ a un nivel de confianza de 95,5 por 100 y factor de corrección. Además de las 62 empresas exportadoras que constituyeron la muestra de nuestro estudio empírico, con el objetivo de recabar una mayor información, se incluyeron a 6 empresas adicionales. Estas empresas incluidas en el estudio, aun no realizaban una actividad exportadora en la actualidad, sin embargo, tenían un alto potencial exportador. Con todo esto, el número total de empresas participantes en nuestro estudio se elevó hasta un total de 68 empresas.

Palabras clave: Comercio electrónico, Tecnologías de la Información y comunicación - TIC, Comercio exterior

\begin{abstract}
The objective of the research on the analysis of electronic commerce in the Junín region and its contribution in international transactions is to determine the characteristics of the exporting companies of the Junín region that have contributed to the implementation of Information and Communication Technologies and trade electronic. The study was developed under the type of applied research, with information from the directory of companies in the Junín region facilitated by the commission for the Promotion of Peru for Exports and Tourism (Promperu Exports- Macro Regional Office for Exportation Center). The research level was descriptive of cross-section. The study had as object of study a population conformed by 104 companies, of which 35 belong to the clothing and decoration sector and, 69 to the agroindustry sector; These in turn directly and indirectly carry out commercial activity abroad; the sample size was 62 companies with an approximate sample error of 8,11 per 100, for $p=q=0,5$ at a confidence level of 95,5 per cent and correction factor. In addition to the 62 export companies that constituted the sample of our empirical study, with the objective of gathering more information, 6 additional companies were included. These companies included in the study, had not yet carried out an export activity at present, nevertheless, they had a high export potential. With all this the total number of companies participating in our study increase to a total of 68 companies.
\end{abstract}

Keywords: E-commerce, Information and Communication Technologies - ICT, Foreign trade.

Historial del artículo:

Recibido, 04 de marzo 2016; aceptado, 10 de mayo de 2016; disponible en línea, 05 de julio de 2016

* Licenciado en Ciencias Empresariales por Esade Business School, Universidad Ramon Llull, Barcelona, España; docente de la Universidad Continental.

Correo: josemariamatasc@gmail.com 


\section{INTRODUCCIÓN}

En el mercado actual caracterizado por la globalización productiva y comercial, la subsistencia de una empresa requiere de un continuo proceso de adaptación al mercado. Por ello, es fundamental para su éxito: poseer un carácter innovador, independientemente del sector al que pertenezca. Es así que se plantea el siguiente problema de investigación ¿Cuáles son las características de las empresas exportadoras de la región Junín que han contribuido a la implantación de las TIC y el comercio electrónico?. Los objetivos son: Determinar las características de las empresas exportadoras de la región Junín que han contribuido a la implantación de las TIC y el comercio electrónico, así como también el nivel de implantación de las TIC y el comercio electrónico de las empresas de la región de Junín y las perspectivas a futuro. La presente investigación se justifica frente a la búsqueda de ventajas competitivas, las empresas no pueden permitirse competir sin una adecuada dotación de herramientas TIC, pues estas permiten a las empresas reducir significativamente los costes de transacción haciendo más rápida la búsqueda de compradores y vendedores, la recopilación de información, etc., (Chen y Seshadri, 2007); es decir, aproxima a oferentes y demandantes facilitando la circulación de bienes y servicios a nivel mundial, con las mejoras productivas y competitivas que ello conlleva. De manera que éstas, se constituyen importantes para que las pequeñas y medianas empresas (PYMES-la mayoría de las empresas en estudio), puedan aprovechar plenamente las oportunidades que les ofrece el mercado (Bernal, Medina, Moral, \& Moral Pajares, 2014). No obstante, la simple dotación de TIC no garantiza la obtención de resultados empresariales óptimos, si no es en combinación con otros factores, como la formación y capacitación de los recursos humanos de la empresa.

Es así que a través de esta investigación se pretende contribuir al estudio del comercio electrónico que las empresas exportadoras desarrollan para mejorar sus ventajas competitivas mediante las TIC.

Las dificultades de esta investigación son que muchas empresas carecen de confianza para brindar la información de manera abierta, es decir que estas empresas como miembros de Promperu facilitaron los datos en vista del estudio propuesto.

\section{MATERIAL Y MÉTODOS}

Para la realización de nuestro estudio partimos de la totalidad de empresas del sector de vestimenta y decoración y del sector de la agroindustria. Posteriormente, fueron seleccionadas para el estudio aquellas empresas que realizan, de manera directa o indirecta, actividad comercial en el exteriorde acuerdo a la influencia respecto al tamaño, antigüedad y rendimiento de la actividad exportadora (Madrid \& Daniel, 2004), resultando 35 empresas exportadoras en el sector de la vestimenta y la decoración y 69 empresas exportadoras del sector de la agroindustria. Estos datos fueron facilitados por Promperú (Comisión de Promoción del Perú para la Exportación y el Turismo).

La población fue de 104 empresas exportadoras, de las cuales 62 aceptaron participar en el estudio,

Tabla 1: Ficha técnica del estudio.

\begin{tabular}{|c|c|}
\hline Población & \\
\hline $\begin{array}{l}\text { Unidades de } \\
\text { muestreo: }\end{array}$ & $\begin{array}{l}\text { Empresas exportadoras del sector de } \\
\text { vestimenta y decoración y del sector } \\
\text { de la agroindustria. }\end{array}$ \\
\hline Población total: & 104 empresas. \\
\hline $\begin{array}{l}\text { Elementos de } \\
\text { muestreo: }\end{array}$ & $\begin{array}{l}\text { Máximos responsable de la } \\
\text { administración de las empresas. }\end{array}$ \\
\hline Alcance: & Región de Junín (Perú) \\
\hline Tiempo: & $\begin{array}{l}\text { Del } 23 \text { de noviembre de } 2015 \text { al } 28 \\
\text { de noviembre de } 2015 \text { en la Selva } \\
\text { Central y del } 1 \text { de diciembre de } 2015 \\
\text { al } 14 \text { de diciembre de } 2015 \text { en el } \\
\text { Valle del Mantaro. }\end{array}$ \\
\hline Tamaño muestral: & 62 empresas. \\
\hline Encuestas válidas: & 62 empresas. \\
\hline $\begin{array}{l}\text { Error muestral } \\
\text { aproximado: }\end{array}$ & $\begin{array}{l}8,11 \text { por } 100 \text {, para } p=q=0,5 \text { a un } \\
\text { nivel de confianza del } 95,5 \text { por } 100 \text { y } \\
\text { factor de corrección. }\end{array}$ \\
\hline
\end{tabular}

constituyendo éstas la muestra; la ficha técnica del estudio se muestra en la tabla 1.

\section{RESULTADOS}

Comenzando por el análisis del origen de las iniciativas innovadoras en la empresa, y como comprobamos en la tabla 2, éstas surgen mayoritariamente de sus órganos decisores (Alderete, 2012). Así, en un 57,35 $\%$ de las ocasiones proceden del gerente. En términos globales, en un $69,13 \%$ de empresas, las iniciativas surgen de manera exclusiva de los órganos decisores o directivos de la empresa, porcentaje al que se debe añadir el 5,88 \% de empresas en las que la iniciativa procede de manera conjunta de empleados y gerente.

En la tabla 3, se muestra que el 30,88 \% de empresas gestiona el sistema TIC a través de empleados con 
Tabla 2: Procedencia de la iniciativa para introducir o incrementar la TIC en la empresa.

\begin{tabular}{lcc}
\hline \multirow{2}{*}{ Respuesta } & \multicolumn{2}{c}{ Empresas Exportadoras } \\
\cline { 2 - 3 } & $N^{\circ}$ empresas & $\%$ \\
\hline Gerente & 39 & 57,35 \\
Otros* & 6 & 8,82 \\
Empleados con conocimientos en TICs & 5 & 7,35 \\
Presidente/ consejo directivo y Gerente & 5 & 7,35 \\
Gerente y Empleados con conocimientos & 4 & 5,88 \\
en TICs & & 5,88 \\
Gerente y otros* & 4 & 4,43 \\
Presidente/ consejo directivo & 3 & 1,47 \\
Presidente/ consejo directivo y Empleados & 1 & 1,47 \\
con conocimientos en Tics & & 100,00 \\
NS/NC & 1 & \\
\hline Total & 68 & \\
\hline
\end{tabular}

* Propietario, gerente de producción, socios, practicante, iniciativa propia.

Fuente: Elaboración propia.

conocimientos a nivel usuario, en algunos casos acuden a especialistas externos, siempre con ayuda interna $(29,42 \%)$ y en otros sin ayuda $(19,12 \%)$. Sin embargo, la opción de gestionar este sistema por empleados con conocimientos avanzados también está presente, siendo $13,23 \%$ las empresas que lo realizan.

La dotación y uso de TIC, permiten a las empresas reducir significativamente los costes de transacción, haciendo más rápida la búsqueda de compradores y vendedores, la recopilación de información, etc., (Chen y Seshadri, 2007). Por ello, abordamos la penetración de estos elementos en su actividad empresarial.

Centrados en el uso de las TIC en las empresas, como vemos en la tabla 4, el $100 \%$ utilizan Internet para enviar y recibir correos electrónicos (Molinillo \& Parra, 2001). la mayoría de empresas del sector utilizan Internet para mantener contacto con los agentes de su entorno. Así, el 97,05\% de empresas se relacionan con sus clientes a través de Internet, el $83,82 \%$ con proveedores, el 73,53 \% realizan gestiones con las administraciones públicas y el 51,47

Tabla 3: Personal encargado de llevar a cabo el sistema TIC en la empresa.

\begin{tabular}{lcc}
\hline \multirow{2}{*}{ Respuesta } & \multicolumn{2}{c}{ Empresas Exportadoras } \\
\cline { 2 - 3 } & $\mathrm{N}^{\circ}$ empresas & $\%$ \\
\hline Empleados con conocimientos a nivel de & 21 & 30,88 \\
usuario en TIC & 20 & 29,42 \\
Expertos externos en TIC, con ayuda interna & 13 & 19,12 \\
Sólo consultores externos & 9 & 13,23 \\
Empleados con conocimientos avanzados & 2 & \\
en TIC & & 2,94 \\
Empleados con conocimientos a nivel de & 1 & 1,47 \\
usuario en TIC y expertos externos en TIC, & & \\
con ayuda interna & 1 & 1,47 \\
Empleados con conocimientos avanzados en & & 1,47 \\
TIC y sólo consultores externos & 1 & 100,00 \\
Empleados con conocimientos a nivel de & 68 & \\
usuario en TIC y sólo consultores externos & & \\
NS/NC & & \\
\hline Total & & \\
\hline
\end{tabular}

Fuente: Elaboración propia. 
$\%$ realizan operaciones bancarias a través de la red. El porcentaje de empresas que utilizan Internet para mantener contacto con su personal es más bajo (un 64,70\%). Este hecho puede justificarse por la reducida dimensión de las empresas analizadas, lo que hace que el personal de la empresa mantenga un contacto directo, con una frecuencia diaria, que hace innecesario el uso de Internet para comunicarse. Por su parte, es muy limitado el uso de Internet para realizar la búsqueda y selección de personal (menos de un $19,12 \%$ de las empresas).

Internet puede ser utilizado para compartir información con socios y clientes, por ejemplo, sobre los niveles de inventario, los planes de producción, etc. Tal y como se observa en la tabla 5, el 69,12\% de empresas intercambian información electrónicamente con proveedores y clientes. El 29,41\% de empresas compartía información solo con sus clientes y solo el 1,47 \% compartía información solo con sus proveedores.

La penetración del comercio electrónico entre las empresas de la región Junín usualmente se realizan a través de las tiendas electrónicas, o tiendas online, así como también mediante las páginas web que facilitan la realización de compras. Analizando las compras realizadas por las empresas que se apoyan en el uso de elementos virtuales (Ponce \& Escanciano, 1997). Como vemos en la tabla 6, más del $72 \%$ de empresas exportadoras analizadas utilizan Internet para realizar sus compras, mientras el $27,94 \%$ aun no lo hace.

No precisamente utilizan tiendas virtuales, sino que, por medio de las páginas web de sus proveedores de insumos y maquinarias, específicamente por la información que aparece en "contáctenos" donde figuran teléfonos y correos electrónicos de la empresa, se realizan pedidos y cotizaciones. A partir del contacto por correo electrónico entre empresa compradora

Tabla 4: Tipo de uso de Internet en la empresa.

\begin{tabular}{|c|c|c|}
\hline \multirow{2}{*}{ Respuesta } & \multicolumn{2}{|c|}{ Empresas Exportadoras } \\
\hline & $\mathrm{N}^{\circ}$ empresas & $\%$ \\
\hline Enviar y recibir correo electrónico & 68 & 100,00 \\
\hline $\begin{array}{l}\text { Relacionarme con mis clientes (redes, } \\
\text { contactos, compras) }\end{array}$ & 66 & 97,05 \\
\hline $\begin{array}{l}\text { Buscar información (proveedores, mercados, } \\
\text { sector productivo, diseños nuevos) }\end{array}$ & 59 & 86,76 \\
\hline $\begin{array}{l}\text { Relacionarme con mis proveedores (redes, } \\
\text { contactos, compras) }\end{array}$ & 57 & 83,82 \\
\hline $\begin{array}{l}\text { Realizar gestiones con la administración } \\
\text { (impuestos, seguridad social, etc.) }\end{array}$ & 50 & 73,53 \\
\hline $\begin{array}{l}\text { Relacionarme con mi personal (comunicados, } \\
\text { invitaciones, documentos) }\end{array}$ & 44 & 64,70 \\
\hline Realizar operaciones bancarias & 35 & 51,47 \\
\hline Búsqueda/selección de personal & 13 & 19,12 \\
\hline Actividades/Aplicaciones específicas del sector & 8 & 11,76 \\
\hline Otras & 1 & 1,47 \\
\hline
\end{tabular}

Fuente: Elaboración propia.

Tabla 5: Uso compartido de información sobre la cadena de suministro de la empresa, con sus proveedores o clientes, de forma regular, en el año 2015.

\begin{tabular}{lcc}
\hline \multirow{2}{*}{ Respuesta } & \multicolumn{2}{c}{ Empresas Exportadoras } \\
\cline { 2 - 3 } & $\mathrm{N}^{\circ}$ empresas & $\%$ \\
\hline $\begin{array}{l}\text { Compartía información con sus proveedores } \\
\text { y clientes }\end{array}$ & 47 & 69,12 \\
Compartía información con sus Clientes & 20 & 29,41 \\
Compartía información con sus proveedores & 1 & 1,47 \\
\hline Total & 68 & 100,00 \\
\hline
\end{tabular}

Fuente: Elaboración propia. 
Tabla 6: Empresas que utilizaban Internet para realizar o iniciar pedidos/reservas de bienes o servicios, en el año 2015.

\begin{tabular}{lcc}
\hline \multirow{2}{*}{ Respuesta } & \multicolumn{2}{c}{ Empresas Exportadoras } \\
\cline { 2 - 3 } & $N^{\circ}$ empresas & $\%$ \\
\hline Sí & 49 & 72,06 \\
No & 19 & 27,94 \\
\hline Total & 68 & 100,00 \\
\hline
\end{tabular}

Fuente: Elaboración propia.

y empresa proveedora se realizan negociaciones de compra (Sohal \& Moss, 2000). En su mayoría las empresas encuestadas utilizan correo electrónico para hacer pedidos de insumos a sus proveedores, bajo esta modalidad las formas de pago son a través de cuentas bancarias.

Analizando las características de las empresas de agroindustria, entre las que destaca las exportadoras de café, más del $80 \%$ de su producción es vendida en materia prima (grano verde) y el resto es transformado en presentaciones de café tostado y molido. Similar es el caso de las empresas exportadoras de maca, en su mayoría exportan harina, harina gelatinizada y chips de maca, el resto de su producción es transformada en cápsulas y licor de maca. En cuanto a las empresas que trabajan con jengibre los productos que exportan son jengibre en fresco y en polvo, productos con mínimo valor agregado.

Observando los resultados de la tabla 7 , el 63,25 \% de empresas manifestó que, del total de sus compras, un porcentaje igual o inferior al $10 \%$ representan las compras iniciadas mediante Internet, esto sería explicado por el hecho de que las empresas no requieren demasiados insumos, por presentar productos con poco valor agregado.

Tabla 7: Porcentaje del importe total de las compras correspondientes a pedidos/reservas de bienes o servicios realizados o iniciados mediante Internet, en el año 2015.

\begin{tabular}{lcc}
\hline Porcentaje de compras a través del & \multicolumn{2}{c}{ Empresas Exportadoras } \\
\cline { 2 - 3 } comercio electrónico & $N^{\circ}$ empresas & $\%$ \\
\hline 0 & 19 & 27,94 \\
$1-5$ & 13 & 19,13 \\
$6-10$ & 11 & 16,18 \\
$11-20$ & 5 & 7,35 \\
$21-30$ & 4 & 5,88 \\
$31-40$ & 2 & 2,94 \\
$41-50$ & 3 & 4,41 \\
$51-60$ & 0 & 0,00 \\
$61-70$ & 2 & 2,94 \\
$71-80$ & 3 & 4,41 \\
$81-90$ & 3 & 4,41 \\
100 & 3 & 4,41 \\
\hline Total & 68 & 100,00 \\
\hline
\end{tabular}

Fuente: Elaboración propia.

Tabla 8: Empresas que conocen los mercados electrónicos como canal de internacionalización.

\begin{tabular}{lcc}
\hline \multirow{2}{*}{ Respuesta } & \multicolumn{2}{c}{ Empresas Exportadoras } \\
\cline { 2 - 3 } & No empresas & $\%$ \\
\hline Sí & 25 & 36,76 \\
No & 43 & 63,24 \\
\hline Total & 68 & 100,00 \\
\hline
\end{tabular}

Fuente: Elaboración propia. 
Tabla 9: Empresas que operan en los mercados electrónicos.

\begin{tabular}{lcc}
\hline \multirow{2}{*}{ Respuesta } & \multicolumn{2}{c}{ Empresas Exportadoras } \\
\cline { 2 - 3 } & $N^{\circ}$ empresas & $\%$ \\
\hline Sí & 12 & 17,65 \\
No & 56 & 82,35 \\
\hline Total & 68 & 100,00 \\
\hline Fuente: Elaboración propia.
\end{tabular}

Los mercados electrónicos pueden ser definidos como intermediarios del comercio electrónico que ponen en contacto a oferentes y demandantes, a través de un espacio virtual, en el que les ofrecen diversas funciones que facilitan la realización de la transacción.

Del estudio que sobre el conocimiento que las empresas de nuestra investigación tienen de estos intermediarios destaca, como podemos ver en la tabla 8, que la mayoría de ellas (un 63,24 \%) no conocen ningún mercado electrónico, frente a un $36,76 \%$ de empresas que sí conocen mercados como: Alibaba, Amazon, Ebay, ETSI, FashionTrade, Linio, Manta, Mercado Libre, Novica, OLX y Solo Stock.
Pasando a analizar el uso que las empresas realizan de los mercados electrónicos, cabe destacar que tan solo 12 de las 25 empresas que decían conocerlos $(48,00 \%)$ está presente en ellos. En términos globales, tan solo un $17,65 \%$ de las empresas exportadoras de la región de Junín se aprovechan de las ventajas de estar presentes en estos mercados.

Como se muestra en la tabla 10, el principal uso es como instrumento para promocionar el producto y contactar con posibles clientes $(66,67 \%$ de empresas que operan en estos mercados). En segundo lugar, los mercados electrónicos son utilizados, por un $33,33 \%$ de las empresas que operan en éstos, para

Tabla 10: Actividades realizadas por las empresas en los mercados electrónicos.

\begin{tabular}{lcc}
\hline \multirow{2}{*}{ Uso de los mercados electrónicos } & \multicolumn{2}{c}{ Empresas Exportadoras } \\
\cline { 2 - 3 } & $\mathrm{N}^{\circ}$ empresas & $\%$ \\
\hline $\begin{array}{l}\text { Son un instrumento para promocionar el } \\
\text { producto y contactar con posibles clientes }\end{array}$ & 8 & 66,67 \\
Contrato de operaciones de venta & 4 & 33,33 \\
Son un canal de información & 3 & 25,00 \\
\hline
\end{tabular}

Base: empresas que operan en los mercados electrónicos (12 empresas).

Fuente: Elaboración propia.

Tabla 11: Razones por las que exportan las empresas.

\begin{tabular}{lcc}
\hline \multirow{2}{*}{ Razones } & \multicolumn{2}{c}{ Empresas Exportadoras } \\
\cline { 2 - 3 } & $N^{\circ}$ empresas & $\%$ \\
\hline $\begin{array}{l}\text { Oportunidad de negocio en los mercados } \\
\text { exteriores }\end{array}$ & 55 & 80,88 \\
$\begin{array}{l}\text { Conseguir una mayor rentabilidad de los } \\
\text { recursos de la empresa }\end{array}$ & 35 & 51,47 \\
$\begin{array}{l}\text { Porque el cliente sea internacionalizado y yo } \\
\text { con él }\end{array}$ & 10 & 14,71 \\
$\begin{array}{l}\text { Reducir el riesgo de dependencia del } \\
\text { mercado nacional }\end{array}$ & 6 & 8,82 \\
Saturación del mercado nacional & 3 & 4,41 \\
Otros & 2 & 2,94 \\
\hline
\end{tabular}

Fuente: Elaboración propia.

Nota: los porcentajes no suman el $100 \%$ debido a la existencia de respuestas múltiples. 
Tabla 12: Tipo de exportación y actitud de la empresa.

\begin{tabular}{lcc}
\hline \multirow{2}{*}{ Respuesta } & \multicolumn{2}{c}{ Empresas Exportadoras } \\
\cline { 2 - 3 } & $N^{\circ}$ empresas & $\%$ \\
\hline En respuesta a pedidos concretos & 31 & 45,59 \\
Regularmente (todos los años) & 27 & 39,70 \\
Con carácter esporádico o circunstancia & 7 & 10,29 \\
Elabora un plan formalizado de exportación & 3 & 4,41 \\
NE & 6 & 8,82 \\
\hline
\end{tabular}

Fuente: Elaboración propia.

cerrar contratos de ventas y un $25 \%$ como canal de información.

\section{Comercio exterior}

"La exportación es una estrategia que facilita el crecimiento empresarial y ampliación del mercado de referencia, y además puede ser un arma de defensa contra los competidores exteriores" (Alvarez, 2003). Es por ello que la presencia en los mercados internacionales de las empresas de la región de Junín permite el aumento de la competitividad de éstas, siendo las oportunidades de negocio en los mercados exteriores la principal razón (el 80,81 \% de empresas señalaba esta razón), seguida del aumento de la rentabilidad (51,47 \%), y "porque el cliente se ha internacionalizado y yo con él", el 14,71\% (tabla 11).

Como vemos en la tabla 12, entre el colectivo encuestado predominan las empresas que exportan en respuesta a pedidos concretos $(45,59 \%)$, seguido por empresas que exportan regularmente todos los años $(39,70 \%)$ y empresas que exportan con carácter esporádico o circunstancia (10,29\%).

En la tabla 13, se observa que Estados Unidos y Alemania son los mercados exteriores preferidos, ya que figuran tanto en el primer, como en el segundo y el tercer destino internacional de las exportaciones para las empresas analizadas.

\section{DISCUSIÓN}

En el actual mercado global productiva y comercial, el uso de las TIC ha generado un cambio de las reglas de la competencia en el mercado (Laundon \& Laundon, 2009), siendo fundamental su uso para que

Tabla 13: Primer país de destino de las exportaciones.

\begin{tabular}{lcc}
\hline \multirow{2}{*}{ Países } & \multicolumn{2}{c}{ Empresas Exportadoras } \\
\cline { 2 - 3 } & $\mathrm{N}^{\circ}$ empresas & $\%$ \\
\hline EE.UU. & 28 & 41,20 \\
Alemania & 7 & 10,30 \\
Francia & 6 & 8,80 \\
Canadá & 5 & 7,40 \\
Colombia & 2 & 2,90 \\
Costa Rica & 2 & 2,90 \\
España & 2 & 2,90 \\
Inglaterra & 2 & 2,90 \\
Suiza & 2 & 2,90 \\
Australia & 1 & 1,50 \\
China & 1 & 1,50 \\
Ecuador & 1 & 1,50 \\
Holanda & 1 & 1,50 \\
Italia & 1 & 1,50 \\
Noruega & 1 & 1,50 \\
NE & 6 & 8,80 \\
\hline Total & 68 & 100,00 \\
\hline
\end{tabular}

Fuente: Elaboración propia. 
las empresas superen el tradicional aislamiento rural tecnológico mediante las estrategias de turbomarketing (Kotler \& Stonich, 1991), facilitando a las empresas el acceso a los mercados internacionales. Así, Internet se convierte en una herrameitna determinante para la expansión de las empresas a nivel mundial (Soumitra \& Beatrix, 2001), permitiéndoles por ejemplo, la reducción significativa de los costes de transacción, haciendo más rápida la búsqueda de compradores y vendedores, la recopilación de información, entre otros. Es así, que resulta especialmente interesante conocer si ha existido una continuidad en el tiempo en la actividad de exportación, el uso de internet en las empresas analizadas, o por el contrario, se tratan de operaciones esporádicas, siendo el resultado del colectivo encuestado en el que predominan las empresas que exportan en respuesta a pedidos concretos un $45 \%$, seguido por empresas que exportan regularmente todos los años $39,70 \%$ y empresas que exportan con carácter esporádico o circunstancia $10,29 \%$.

Consideramos que el porcentaje de empresas que aún no realizan actividades comerciales con el exterior de manera continuada tiene relación con la escasa experiencia en estos mercados. El aumento de la experiencia de las empresas en este comercio lleva a éstas a reducir la incertidumbre y, por tanto, se produce en afianzamiento en el mercado exterior.

Entre las conclusiones, se tiene que, las empresas analizadas tienen en la mayoría de los casos una antigüedad inferior a los 10 años, por lo que podríamos calificarlas como jóvenes. Existe cierto apoyo de la dirección a las iniciativas propuestas por los trabajadores de la empresa. Una posible respuesta a esta confianza puede residir en el tamaño de las empresas, que como hemos visto en apartados anterior es pequeño, prevaleciendo las Pymes y con un elevado número de microempresas.

El máximo responsable de la entidad, en la mayoría de los casos es el Gerente, quien promueve la introducción o incremento elementos TIC en la empresa. Siendo el $38 \%$ empleados con conocimientos a nivel de usuario en TIC, por lo que se recomienda que estos deben de ser impulsados por los gerentes de la empresa para su involucramiento en el desarrollo de TIC.

El uso de las TIC, permiten a las empresas reducir significativamente los costes de transacción haciendo más rápida la búsqueda de compradores y vendedores, asimismo facilitan a las empresas el acceso a los mercados internacionales. Una de las exigencias que se caracteriza en las empresas innovadora que utilizan TIC'S es el elevado nivel de uso de Internet para mantener contacto con su entorno (clientes, proveedores o administración públicas), aprovechando así la capacidad de Internet para acortar las distancias entre la empresa y sus stakeholders y de reducir el tiempo de las operaciones con las administraciones públicas o el sector bancario.

\section{REFERENCIAS BIBLIOGRÁFICAS}

Alderete, M. (2012). Investigaciones comerciales. En M. Alderete, From traditional transactions to B2B: a contract theoretical analysis (págs. 17-26). Texas: B2B.

Alvarez, A. (2003). Continuidad y cambio en la España urbana. En A. Alvarez, Continuidad y cambio en la España urbana en el período de entreguerras (pág. 216). España: netbibio.

Bernal, E., Medina, V., Moral, A., \& Moral Pajares, E. (2014). Factores determinantes para la exportación en las empresas cooperativas oleícolas andaluzas. Ciriec-España, 81.

Cheng, Y. J., \& Seshadri, S. (2007). Impacts of relevant factors on versioning. En Y. J. Cheng, \& S. Seshadri, Impacts of relevant factors on versioning (pág. 1002). España: Ager.

Kotler, P., \& Stonich, P. (1991). Turbo Marketing Through Time Compression. En P. Kotler, Turbo Marketing (pág. 239). U.S.A.: MCB UP .

Laundon, J., \& Laundon, K. (2009). Essentials of Management Information Systems. En J. Laundon, \& K. Laundon, Essentials of Management Information Systems (pág. 365). U.S.A.: Pearson.

Madrid, A., \& Daniel, G. (2004). Actividad exportadora. En A. Madrid, \& G. Daniel, 6. MADRID, A. y GARCÍA, D. (2004): "Influencia del tamaño, la antigüedad y el rendimiento sobre la intensidad exportadora de la pyme industrial española", (pág. 294). España: Lond.

Molinillo, S., \& Parra, F. (2001). Centros comerciales virtuales. En S. Molinillo, \& F. Parra, E-Business y comercio electronico. Un enfoque estrategico RAMA (págs. 26-56). Madrid: RA-MA.

Ponce, J., \& Escanciano, J. (1997). Comercio Electronico: evolución empresarial. Boletin Fundesco, 188-189.

Sohal, A., \& Moss, S. (2000). Using information technology productively. En A. Sohal, \& S. Moss, International Journal of Technology Management (págs. 340-344). Texas: Technology Management.

Soumitra, D., \& Beatrix , B. (2001). Business Transformation on the Internet: Results from the 2000 Study. En D. Soumitra, \& B. Beatrix, Business Transformation on the Internet (pág. 462). España: European Management Jorunal. 